07

\title{
Расчет передаточных функций многослойных биотканей в задачах коррекции их спектров флуоресценции
}

\author{
(C) С.А. Лысенко \\ Белорусский государственный университет, \\ 220030 Минск, Беларусь \\ e-mail: lisenko@bsu.by
}

Поступила в редакцию 16.05.2017 г.

В окончательной редакции 08.09.2017 г.

\begin{abstract}
Разработан метод быстрого расчета потока вынужденной флуоресценции многослойной оптически плотной среды с неоднородным распределением в ней флуорофора. Световое поле в среде на длине волны возбуждения флуоресценции представлено суперпозицией падающего коллимированного, падающего диффузного и отраженного диффузного потоков. Для описания светового поля в среде на длине волны испускания флуоресценции используется двухпотоковое приближение. Потоки в соседних элементарных слоях среды и на ее поверхности связаны простыми матричными операторами, полученными сочетанием инженерных методов теории переноса излучения с приближением однократного рассеяния. Расчеты потоков флуоресценции четырехслойной биоткани, возбуждаемых и регистрируемых в диапазоне $400-800 \mathrm{~nm}$, сопоставлены с результатами их моделирования методом Монте-Карло с расхождением на уровне $1 \%$. Исследовано влияние среды распространения излучения на спектры флуоресценции 5-АЛК-индуцированного протопорфирина IX, регистрируемые с поверхности кожи человека, и предложена методика их коррекции на основе измерений и количественного анализа спектра диффузного отражения кожи.
\end{abstract}

DOI: $10.21883 /$ OS.2018.01.45368.114-17

\section{Введение}

Методы флуоресцентного исследования биотканей, основанные на регистрации и анализе спектров флуоресценции эндогенных (естественных) и экзогенных (специально введенных) биомаркеров, широко используются в современной медицине для неинвазивного выявления опухолей и уточнения их границ [1-3]. Имеется ряд доклинических и клинических данных, убедительно показывающих высокую эффективность использования природных флуорофоров биоткани (коллагена, эластина, $\mathrm{NADH}$, флавинов, порфиринов и др.) для контроля метаболической активности клеток, оценки степени ишемии ткани и ранней диагностики сахарного диабета [3-5]. Однако для разработки и использования в клинической практике объективных диагностических критериев, основанных на связи спектра флуоресценции биоткани с происходящими в ней деструктивными и иными процессами, необходимо иметь возможность извлечения из измеряемого спектра информации о концентрациях флуоресцирующих биомаркеров, чувствительных к этим процессам.

Поскольку биоткани являются сильно мутными средами, многократно рассеивающими и поглощающими как возбуждающее, так и флуоресцентное излучение, то результирующий спектр флуоресценции, измеряемый диагностическим прибором, может быть существенно искажен по форме и интенсивности в сравнении с фактическим спектром флуоресценции биомаркера внутри среды $[3,6]$. Под последним здесь и далее понимается произведение концентрации $C_{f}$, коэффициента поглоще- ния $\varepsilon_{f}\left(\lambda_{e x}\right)$ и квантового выхода флуоресценции $Q_{f}\left(\lambda_{e m}\right)$ биомаркера на длинах волн его возбуждения $\lambda_{\text {ex }}$ и эмиссии $\lambda_{\text {em }}$.

Известен ряд методов компенсации эффектов поглощения и рассеяния света в мутных средах при измерениях их сигналов флуоресценции. В эмпирических методах результат измерений представляется в виде сигнала флуоресценции $F\left(\lambda_{e x}, \lambda_{e m}\right)$, нормированного на эмпирическую функцию отражательных способностей среды $R$ (измеряемых при той же геометрии, что сигнал $F$ ) на длинах волн возбуждения $\lambda_{e x}$ и испускания $\lambda_{e m}$ флуоресценции [5-8]. Параметры корректирующей функции определяют путем минимизации разброса результатов измерений для тестовых сред с различными оптическими параметрами и одинаковым содержанием флуорофора [5-7]. В работе [8] на основе экспериментальных исследований и численного моделирования переноса излучения в однородной среде показано, что при измерении сигналов флуоресценции и отражения на двух оптимальных и различающихся расстояниях от облучаемой площадки отношение этих сигналов слабо подвержено влиянию поглощения и рассеяния света в среде. Однако данный метод не учитывает различие оптических параметров среды на длинах волн $\lambda_{e x}$ и $\lambda_{e m}$, накладывает жесткие ограничения на конструкцию измерительного устройства и требует калибровочных измерений для сред с известными концентрациями флуорофора.

Методы коррекции спектров флуоресценции, использующие для определения передаточных функций биотканей численные и аналитические модели переноса в них 
излучения, отличаются наибольшей универсальностью и простотой экспериментальной реализации. Точность таких методов определяется точностью описания светового поля в среде, моделирующей исследуемую ткань, и соответствием модели реальному объекту. Эталонным методом теории переноса излучения считается метод Монте-Карло (МК) [9,10], основанный на моделировании траекторий большого количества фотонов в среде и учете вклада каждого фотона в сигнал, измеряемый в эксперименте. Метод МК потенциально позволяет решать задачу коррекции спектров флуоресценции биотканей с детальным учетом их строения и оптических свойств [11-16], однако требует больших вычислительных затрат, неприемлемых для рутинной медицинской диагностики.

Хорошую альтернативу методу МК в расчетах сигналов отражения и флуоресценции однородных сред представляют методы, использующие теорию миграции фотонов [17,18]. Основываясь на этой теории, корректирующая функция для сигнала флуоресценции рассчитывается в простом аналитическом виде при следующих предположениях: 1) мощность фотона при каждом акте рассеяния уменьшается пропорционально альбедо однократного рассеяния среды; 2) вероятность регистрации фотона прибором экспоненциально уменьшается с увеличением количества испытанных им актов рассеяния; 3) обратно рассеянные фотоны и фотоны флуоресценции, регистрируемые одним и тем же прибором, проходят одинаковый путь в среде. К недостаткам этих методов можно отнести невозможность работы с многослойными средами и необходимость эмпирического определения аппаратурно-зависящих параметров расчетной формулы.

Аналитические модели переноса излучения, основанные на законе Ламберта-Бера $[19,20]$, двухпотоковой теории Кубелки-Мунка $[21,22]$ и диффузионном приближении [23-26], применимы для сред с альбедо однократного рассеяния, близким к 0 или 1 (доля рассеяния в общем ослаблении света). При корректном сочетании этих моделей, например при использовании закона Ламбера-Бера для тонкого эпителия и диффузионного приближения для сильно рассеивающей и слабо поглощающей свет стромы, результаты расчетов достаточно хорошо согласуются с более строгими моделями [27]. Однако в общем случае к результатам расчета характеристик переноса излучения в биотканях на основе известных аналитических моделей следует относиться с большой осторожностью, поскольку границы их применимости весьма условны.

В ряде случаев выражения для передаточных функций среды, получаемые в рамках того или иного приближения теории переноса излучения, используются для аппроксимации результатов моделирования методом МК. На этой основе построены аппроксимационные модели сигналов флуоресценции однородной полубесконечной среды с равномерным [28,29] и слоисто-неоднородным распределением в ней флуорофора [30]. Модели [28,30] соответствуют одномерной геометрии измерений, при которой в апертуру приемника попадают фотоны со всей поверхности среды. Модель [29] применима в тех случаях, когда для возбуждения и регистрации флуоресценции используется одно и то же оптическое волокно, подводимое вплотную к исследуемому объекту.

В настоящей работе предложен метод расчета потока флуоресценции с поверхности многослойной оптически плотной среды, не требующий длительных вычислений и обеспечивающий хорошее согласие результатов расчета с методом МК. В его основе лежит представление среды последовательностью тонких слоев и матричные уравнения баланса направленных и диффузных потоков в слоях среды и на ее поверхности. При этом для связи световых потоков на границах элементарных слоев не требуется введения каких-либо эмпирических параметров, не связанных напрямую с фундаментальными оптическими свойствами среды, как, например, в двухпотоковой теории Кубелки-Мунка и четырехпотоковой модели переноса излучения [31]. Вместо этого выбором оптической толщины элементарных слоев обеспечивается возможность вычисления их коэффициентов пропускания и отражения при направленном и диффузном освещении по аналитическим формулам асимптотической теории переноса излучения и приближения однократного рассеяния. Эти приемы использовались нами ранее в задачах персонифицированной фототерапии биотканей для оценок эффективности доставки излучения в слои ткани различной глубины [32-34]. Ниже предпринята попытка учета собственного излучения элементарных слоев среды вследствие их возбуждения на смещенной длине волны.

Возможности предлагаемого метода демонстрирутся на примерах коррекции спектров флуоресценции кожи человека. В качестве флуоресцирующей субстанции рассматривается 5-АЛК-индуцированный протопорфирин IX (ПП IX), широко используемый для флуоресцентной диагностики и фотодинамической терапии рака [35]. Оптические параметры кожи, необходимые для расчета ее передаточных функций, определяются на основе решения обратной задачи спектроскопии диффузного отражения с использованием аппроксимационного аналога метода МК [36].

\section{Метод расчета потока флуоресценции многослойной среды}

Рассмотрим одномерную задачу флуоресценции, в которой плоскопараллельная многослойная среда освещается пучком лучей малого диаметра (в пределе бесконечно малого), а приемник захватывает световой поток со всей светящейся поверхности среды, либо когда среда освещается широким пучком лучей (в пределе - бесконечно широким), а приемник регистрирует поток флуоресценции из центра освещаемой области. Метрологически правильный вариант проведения таких 
измерений предполагает использование интегрирующей сферы, однако для исследования биотканей in vivo более удобны бесконтактные измерительные устройства, в которых для регистрации сигнала флуоресценции используются ПЗС-камера $[37,38]$ или волокнооптический датчик, отведенный от исследуемого участка ткани на некоторое расстояние [39]. Достоинства таких измерителей - отсутствие искажающего нажима на ткань, возможность устранения вклада зеркального отражения света в регистрируемый сигнал и простота калибровки.

Флуоресценция представляет собой двухступенчатый процесс, включающий возбуждение молекул флуорофора на длине волны $\lambda_{e x}$ и испускание ими излучения с длиной волны $\lambda_{e m}$, смещенной в красную область спектра. Величина сигнала флуоресценции, регистрируемого с поверхности среды, определяется следующим выражением:

$$
\begin{aligned}
F\left(\lambda_{e x}, \lambda_{e m}\right)= & \sum_{f=1}^{N_{f}} \int_{0}^{z_{\max }} C_{f}(z) \varepsilon_{f}\left(\lambda_{e x}\right) \\
& \times Q_{f}\left(\lambda_{e m}\right) E\left(z, \lambda_{e x}\right) T\left(z, \lambda_{e m}\right) d z,
\end{aligned}
$$

где $C_{f}(z)$ - концентрация флуорофора $f$ на глубине среды $z, \varepsilon_{f}\left(\lambda_{e x}\right)$ и $Q_{f}\left(\lambda_{e m}\right)$ - его показатель поглощения и квантовый выход флуоресценции, $N_{f}$ - общее количество флуорофоров среды, поглощающих на $\lambda_{e x}$ и излучающих на $\lambda_{e m}, E\left(z, \lambda_{e x}\right)-$ поток возбуждающего излучения на глубине $z, T\left(z, \lambda_{e m}\right)-$ функция, характеризующая передачу мощности излучения от изотропного точечного источника на глубине $z$ к поверхности среды, $z_{\max }$ - толщина среды.

Задача расчета $E\left(z, \lambda_{\text {ex }}\right)$ достаточно подробно рассмотрена в [32]. Световое поле в среде представляется суперпозицией трех потоков - падающего коллимированного $I_{n}$, падающего диффузного $I_{n}^{*}$ и отраженного диффузного $J_{n}^{*}$. Для связи световых потоков внутри и на границах среды она мысленно разбивается на элементарные слои с оптической толщиной $\Delta \tau=0.04-0.05$ $(\Delta \tau-$ величина, стоящая в экспоненте закона Бугеpa, - произведение показателя ослабления и толщины слоя). Для каждого слоя в матричном виде записывается система линейных уравнений, выражающих баланс отраженного, пропущенного и поглощенного слоем потоков при его освещении сверху и снизу. Последовательно перемножая матрицы элементарных слоев $\Delta M_{n}$, начиная с самого верхнего (ближайшего к освещаемой поверхности среды), можно световые потоки на оптической глубине $\tau_{n}=n \Delta \tau$ выразить через аналогичные потоки на ее поверхности:

$$
\left(\begin{array}{lll}
I_{n} & I_{n}^{*} & J_{n}^{*}
\end{array}\right)^{T}=M_{n}\left(\begin{array}{lll}
I_{0} & I_{0}^{*} & J_{0}^{*}
\end{array}\right)^{T}
$$

где $I_{0}$ и $I_{0}^{*}$ - падающие на среду коллимированный и диффузный потоки, $J_{0}^{*}$ - отраженный средой диффузный поток,

$$
\begin{aligned}
M_{n} & =\prod_{k=1}^{n} \Delta M_{k} \\
& =\prod_{k=1}^{n}\left(\begin{array}{ccc}
t & 0 & 0 \\
\left(f_{k} t_{k}^{*}-b_{k} r_{k}^{*}\right) / t_{k}^{*} & \left(\left(t_{k}^{*}\right)^{2}-\left(r_{k}^{*}\right)^{2}\right) / t_{k}^{*} & r_{k}^{*} / t_{k}^{*} \\
-b_{k} / t_{k}^{*} & -r_{k}^{*} / t_{k}^{*} & 1 / t_{k}^{*}
\end{array}\right),
\end{aligned}
$$

$n=1, \ldots, N, N$ - общее количество элементарных слоев, формирующих среду, $t=\exp \left(-\Delta \tau / \mu_{0}\right)-$ пропускание нерассеянного света элементарным слоем, $\mu_{0}-$ косинус угла падения излучения, $f_{k}$ и $b_{k}-$ коэффициенты рассеяния слоем излучения соответственно в верхнюю и нижнюю полусферы при его освещении в направлении $\mu_{0}, r_{k}^{*}$ и $t_{k}^{*}-$ коэффициенты отражения и пропускания слоя при его диффузном освещении.

Коэффициенты $r_{k}^{*}, t_{k}^{*}, f_{k}$ и $b_{k}$ рассчитываются в зависимости от оптических параметров слоя на глубине $\tau_{k}$ с использованием инженерных методов теории переноса излучения [40] и приближения однократного рассеяния [41]:

$$
\begin{gathered}
r_{k}^{*}=\frac{\operatorname{sh}\left[\gamma_{k} \Delta \tau\right]}{\operatorname{sh}\left[\gamma_{k} \Delta \tau+(4 / 3) \gamma_{k} /\left(1-g_{k}\right)\right]}, \\
t_{k}^{*}=\frac{\operatorname{sh}\left[(4 / 3) \gamma_{k} /\left(1-g_{k}\right)\right]}{\operatorname{sh}\left[\gamma_{k} \Delta \tau+(4 / 3) \gamma_{k} /\left(1-g_{k}\right)\right]}, \\
f_{k}=\frac{\omega_{k}}{2} \int_{0}^{1} \frac{h_{k}\left(\mu_{0}, \mu\right)}{\mu-\mu_{0}} \\
\times\left\{\exp \left(-\frac{\Delta \tau}{\mu}\right)-\exp \left(-\frac{\Delta \tau}{\mu_{0}}\right)\right\} \mu d \mu, \\
b_{k}=\frac{\omega_{k}}{2} \int_{0}^{1} \frac{h_{k}\left(\mu_{0},-\mu\right)}{\left(\mu_{0}+\mu\right)} \\
\times\left\{1-\exp \left[-\Delta \tau\left(\frac{1}{\mu_{0}}+\frac{1}{\mu}\right)\right]\right\} \mu d \mu,
\end{gathered}
$$

где $\omega_{k}$ - альбедо однократного рассеяния, $g_{k}-$ средний косинус индикатрисы рассеяния, $\gamma_{k}=$ $=\sqrt{3\left(1-\omega_{k}\right)\left(1-g_{k}\right)}-$ безразмерный глубинный показатель ослабления, $h_{k}\left(\mu, \mu_{0}\right)$ - азимутальноусредненная индикатриса рассеяния слоя, которая для однопараметрической модели индикатрисы Хеньи-Гринштейна может быть представлена в следующем виде:

$$
\begin{aligned}
& h_{k}\left(\mu_{0}, \mu\right)=\frac{1-g_{k}^{2}}{2 \pi} \\
& \times \int_{0}^{2 \pi}\left(1+g_{k}^{2}-2 g_{k}\left(\mu \mu_{0}+\sqrt{1-\mu^{2}} \sqrt{1-\mu_{0}^{2}} \cos \varphi\right)\right)^{-3 / 2} d \varphi .
\end{aligned}
$$


При малой оптической толщине слоя $\Delta \tau \sim 0.001$ рассеяние света в нем является преимущественно однократным, поэтому уменьшением $\Delta \tau$ можно добиться сколь угодно высокой точности вычисления коэффициентов $f_{k}$ и $b_{k}$ по формулам (6), (7), вытекающим из стационарного уравнения переноса неполяризованного излучения для плоскопараллельной горизонтально однородной среды [41] (когда интегральным слагаемым функции источников можно пренебречь по сравнению со слагаемым, описывающим однократное рассеяние падающего на среду излучения). Однако формулы (4), (5) изначально были получены для оптически толстых слоев [40] в предположении, что в них реализуется так называемый „асимптотический режим“, когда угловая диаграмма интенсивности излучения не зависит от оптической глубины. Тем не менее, как показано нами в [32], при значениях $\Delta \tau=0.04-0.05$ и при большой суммарной оптической толщине слоев удается достичь приемлемого компромисса между двумя используемыми приближениями. Основные погрешности метода связаны с недостаточно точным описанием переноса излучения в приповерхностных слоях среды, через которые свет распространяется преимущественно в виде направленного пучка. Однако вследствие многократных переотражений света между поверхностью и слоями среды эти погрешности несущественно сказываются на распределении суммарного потока в среде и быстро убывают с уменьшением ее анизотропии рассеяния.

Потоки $I_{0}$ и $I_{0}^{*}$ в (2) определяются условиями освещения среды. Отраженный средой поток $J_{0}^{*}$ находится из условия равенства нулю восходящего потока $J_{N}^{*}$ для самого глубокого слоя среды $(n=N)$. Перепад показателя преломления на границе среды создает бесконечно убывающую геометрическую прогрессию потоков, многократно переотраженных между средой и ее поверхностью, с учетом которых выражение для результирующего потока на оптической глубине среды $\tau_{n}$, при ее освещении направленным пучком лучей, имеет следующий вид [32]:

$$
\begin{aligned}
E\left(\tau_{n}\right)= & E_{0}\left(1-\rho_{s}\right)\left[\sum_{i=1}^{3}\left(m_{i, 1}^{(n)}+R_{0} m_{i, 3}^{(n)}\right)\right. \\
& \left.+\frac{\rho_{s}^{*} R_{0}}{1-\rho_{s}^{*} R_{0}^{*}} \sum_{i=1}^{3}\left(m_{i, 2}^{(n)}+R_{0}^{*} m_{i, 3}^{(n)}\right)\right],
\end{aligned}
$$

где $E_{0}-$ освещенность среды, $\rho_{s}-$ коэффициент отражения направленного потока от наружной стороны поверхности среды, $\rho_{s}^{*}-$ коэффициенты отражения диффузного потока от внутренней стороны поверхности среды, $R_{0}=-m_{3,1}^{(N)} / m_{3,3}^{(N)}$ и $R_{0}^{*}=-m_{3,2}^{(N)} / m_{3,3}^{(N)}-$ коэффициенты диффузного отражения (КДО) среды с прозрачной поверхностью при направленном и диффузном освещении соответственно, $m_{i, j}^{(n)}$ - элементы матрицы (3).

Рассмотрим теперь вторую часть решаемой задачи расчет потока флуоресценции на поверхности среды при заданных распределениях концентрации флуорофора и потока возбуждающего излучения по глубине среды. Поскольку флуоресцентная эмиссия в элементарном слое среды изотропна, то световое поле в среде на длине волны ее флуоресценции $\lambda_{e m}$ можно представить в виде суммы восходящего $J_{n}^{*}$ и нисходящего $I_{n}^{*}$ диффузных потоков. Запишем уравнения, связывающие световые потоки в соседних элементарных слоях среды:

$$
\begin{aligned}
& I_{n}^{*}=I_{n-1}^{*} t_{n}^{*}+J_{n}^{*} r_{n}^{*}+B_{n} \Delta z_{n} / 2, \\
& J_{n-1}^{*}=I_{n-1}^{*} r_{n}^{*}+J_{n}^{*} t_{n}^{*}+B_{n} \Delta z_{n} / 2,
\end{aligned}
$$

где $B_{n}=E\left(z, \lambda_{e x}\right) C_{f}\left(z_{n}\right) \varepsilon_{f}\left(\lambda_{e x}\right) Q_{f}\left(\lambda_{e m}\right)-$ функция источников; $C_{f}\left(z_{n}\right)$ - концентрация флуорофора на глубине $z_{n} ; \Delta z_{n}=\Delta \tau /\left(\mu_{a}\left(z_{n}, \lambda_{e x}\right)+\mu_{s}\left(z_{n}, \lambda_{e x}\right)\right)$ - шаг пространственной дискретизации по $z$, соответствующий равномерной сетке по $\tau$ в рассмотренной выше задаче расчета $E\left(\tau_{n}, \lambda_{e x}\right) ; \mu_{a}(z)$ и $\mu_{s}(z)$ - профили показателей поглощения и рассеяния среды; $r_{n}^{*}$ и $t_{n}^{*}-$ коэффициенты диффузного отражения и пропускания элементарного слоя $\Delta z_{n}$, рассчитываемые по формулам (4) и (5).

Представим систему уравнений (10) в матричной форме:

$$
\begin{aligned}
\left(\begin{array}{c}
J_{n}^{*} \\
I_{n}^{*}
\end{array}\right)= & \Delta H_{n}\left(\begin{array}{c}
J_{n-1}^{*} \\
I_{n-1}^{*}
\end{array}\right)+\Delta \mathbf{b}_{n} \\
= & \left(\begin{array}{cc}
1 / t_{n}^{*} & -r_{n}^{*} / t_{n}^{*} \\
r_{n}^{*} / t_{n}^{*} & t_{n}^{*}-\left(r_{n}^{*}\right)^{2} / t_{n}^{*}
\end{array}\right)\left(\begin{array}{c}
J_{n-1}^{*} \\
I_{n-1}^{*}
\end{array}\right) \\
& +\frac{B_{n} \Delta z_{n}}{2}\left(\begin{array}{c}
-1 / t_{n}^{*} \\
1-r_{n}^{*} / t_{n}^{*}
\end{array}\right)
\end{aligned}
$$

где $\Delta H_{n}$ - передаточная матрица для слоя $\Delta z_{n}, \Delta \mathbf{b}_{n}-$ вектор, характеризующий генерацию собственного излучения слоем $\Delta z_{n}$. Уравнение (11) позволяет рекурсивно вычислять световые потоки в слоях среды, начиная с самого верхнего слоя, и тем самым выразить потоки на глубине $z_{n}=\sum_{k=1}^{n} \Delta z_{k}$ через восходящий $J_{0}^{*}$ и нисходящий $I_{0}^{*}$ потоки у поверхности среды:

$$
\begin{aligned}
\left(\begin{array}{c}
J_{n}^{*} \\
I_{n}^{*}
\end{array}\right) & =H_{n}\left(\begin{array}{c}
J_{0}^{*} \\
I_{0}^{*}
\end{array}\right)+\mathbf{b}_{n} \\
& =\left(\prod_{k=1}^{n} \Delta H_{k}\right)\left(\begin{array}{c}
J_{0}^{*} \\
I_{0}^{*}
\end{array}\right)+\sum_{k=1}^{n}\left(\prod_{l=k+1}^{n} \Delta H_{l}\right) \Delta \mathbf{b}_{k},
\end{aligned}
$$

где $H_{n}$ и $\mathbf{b}_{n}-$ передаточная матрица и вектор источников для совокупности последовательных слоев $\Delta z_{1}, \ldots, \Delta z_{n}$.

Полагая, что восходящий поток $J_{N}^{*}$ для самого глубокого слоя среды $\Delta z_{N}$ равен нулю, из (12) получаем уравнение для потоков у поверхности среды

$$
h_{1,1}^{N} J_{0}^{*}+h_{1,2}^{N} I_{0}^{*}+b_{1}^{N}=0,
$$

где $h_{i, j}^{N}$ и $b_{i}^{N}$ - элементы матрицы $H_{N}$ и вектора $\mathbf{b}_{N}$. При отсутствии перепада показателя преломления на 
границе раздела сред отраженный поток $I_{0}^{*}$ не возникает и из (13) можно получить значение восходящего потока флуоресценции: $J_{0}^{*}=-b_{1}^{N} / h_{1,1}^{N}$. Отражение света от поверхности среды создает бесконечную последовательность выходящих из среды потоков, образующих убывающую геометрическую прогрессию со знаменателем $\rho_{s}^{*} R_{0}^{*}$, где $\rho_{s}^{*}-$ френелевский коэффициент отражения поверхности при ее освещении изнутри диффузным потоком, $R_{0}^{*}$ - КДО среды при диффузном освещении. Коэффициент $R_{0}^{*}$ можно найти из (13) при $b_{1}^{N}=0$ (что означает отсутствие в среде источников излучения): $R_{0}^{*}=J_{0}^{*} / I_{0}^{*}=-h_{1,2}^{N} / h_{1,1}^{N}$. Сумма всех потоков, выходящих с поверхности среды, дает величину искомого сигнала флуоресценции:

$$
F=\left(1-\rho_{s}^{*}\right) J_{0}^{*} /\left(1-\rho_{s}^{*} R_{0}^{*}\right),
$$

Таким образом, предлагаемый метод позволяет в полуаналитическом виде рассчитывать поток флуоресцентного свечения плоскопараллельной многослойной оптически плотной среды при неоднородном распределении в ней флуорофора. Последовательность выполняемых операций включает вычисление по формулам (4)-(8) коэффициентов $r_{k}^{*}, t_{k}^{*}, f_{k}, b_{k}$ на длинах волн $\lambda_{e x}$ и $\lambda_{e m}$ для каждого слоя среды с отличающимися оптическими параметрами, а также перемножение и сложение передаточных матриц размерностей $(2 \times 2)$ и $(3 \times 3)$ для элементарных слоев, на которые разбивается исследуемая среда. Такие вычисления не являются времязатратными и на современной вычислительной технике выполняются практически в реальном времени.

\section{Верификация метода}

Сопоставим расчеты передаточных функций биотканей передоложенным методом с результатами их моделирования методом МК и с данными литературных источников. Вначале рассмотрим простейшую модель однородной среды и результаты независимых расчетов характеристик переноса в ней излучения, опубликованные в $[28,42]$.

В работе [28] для расчета функции выхода флуоресценции с глубины однородной полубесконечной среды предложено использовать формулы диффузионного приближения с эмпирическими параметрами, полученными аппроксимацией результатов численного моделирования светового поля в среде. Для их практического использования КДО среды на длинах волн $\lambda_{e x}$ и $\lambda_{e m}$ должен быть измерен или рассчитан (по известным значениям показателя поглощения $\mu_{a}$, показателя рассеяния $\mu_{s}$ и среднего косинуса индикатрисы рассеяния среды $g$ ). Данные формулы хорошо согласуются с результатами прямых измерений световых потоков внутри и вне искусственных образцов однородной биологической ткани [28], что позволяет использовать их для верификации новых методов решения одномерной задачи флуоресценции. Результаты такой верификации для метода, описанного вы-

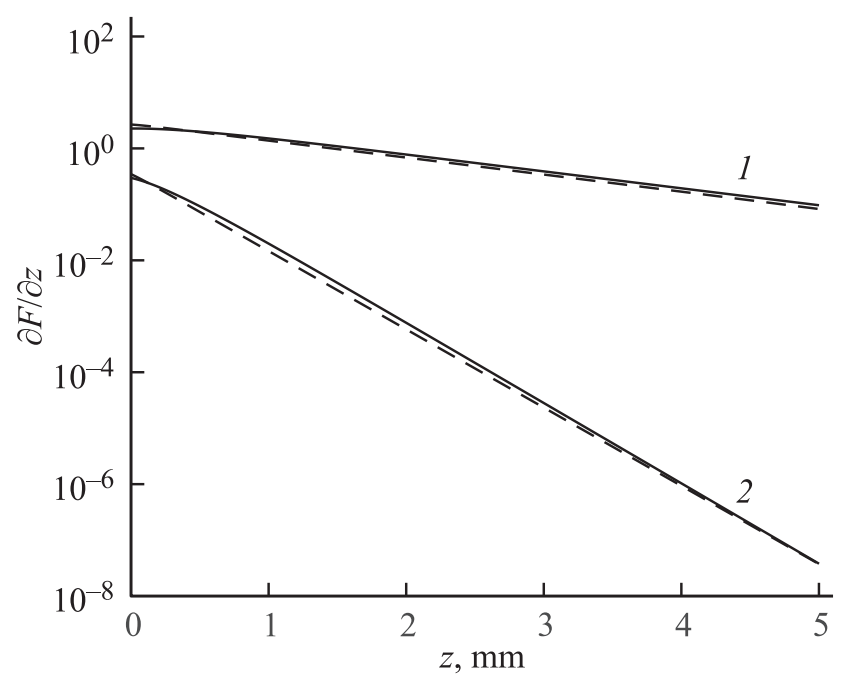

Рис. 1. Вклад различных слоев однородной полубесконечной среды в поток флуоресценции, регистрируемый с еe поверхности: расчет по формулам из [28] (сплошные линии) и разработанным методом (штриховые линии) при $\mu_{a}=0.04 \mathrm{~mm}^{-1}, \quad \mu_{s}=10 \mathrm{~mm}^{-1}, \quad g=0.9, \quad \eta=1.38 \quad$ (1) и $\mu_{a}=0.8 \mathrm{~mm}^{-1}, \mu_{s}=10 \mathrm{~mm}^{-1}, g=0.9, \eta=1.38(2)$.

ше, приведены на рис. 1. Сравниваемая характеристика переноса излучения - дифференциальная передаточная функция среды $\partial F / \partial z$, определяющая вклад элементарного слоя среды на глубине $z$ в детектируемый сигнал флуоресценции $F$. Расчет выполнен в предположении одинаковости оптических параметров среды на длинах волн возбуждения и испускания флуоресценции и при показателе преломления среды $\eta=1.38$, соответствующем формулам [28]. КДО среды вычислялся по предложенной нами ранее аппроксимационной формуле [43], максимальная погрешность которой в области значений оптических параметров биотканей не превышает 5\%. Видно, что распределения $\partial F / \partial z$, рассчитанные двумя методами, достаточно хорошо согласуются между собой, а соответствующие им сигналы $F$ отличаются на 5 и $9 \%$ при $\mu_{a}=0.04$ и $0.8 \mathrm{~mm}^{-1}$ соответственно, что с учетом погрешностей аппроксимационных формул $[28,43]$ является вполне допустимым.

Расчеты сигналов флуоресценции однородной среды также сопоставлены с независимыми данными, полученными в работе [42] тремя вариантами метода МК (таблица). Следует заметить, что в ускоренном варианте метода МК [42] не учитывается изменение расходимости пучка лучей при его прохождении через границу раздела сред (формула (6) в [42]), что приводит к отличию сравниваемых данных в $\eta^{2}$ раз. В остальном расхождение между результатами наших расчетов и [42] не превышает 5\%.

В качестве примера многослойной биоткани рассмотрим кожу человека. Представим ее плоскопараллельной горизонтально-однородной средой, состоящей из четыpex слоев: эпидермиса (1), папиллярной дермы (2), 
Поток флуоресценции с поверхности однородной среды толщиной $5 \mathrm{~mm}$, нормированный на поток возбуждающего излучения*

\begin{tabular}{c|c|c}
\hline $\begin{array}{c}\text { Изменяемый } \\
\text { параметр среды }\end{array}$ & $\begin{array}{c}\text { Данные [42], } \\
\text { скорректированные на } \eta^{2}\end{array}$ & $\begin{array}{c}\text { Расчет } \\
\text { разработанным методом }\end{array}$ \\
\hline- & $0.0330-0.0341$ & 0.0332 \\
$\mu_{a}\left(\lambda_{e x}\right)=0.1 \mathrm{~mm}^{-1}$ & $0.0257-0.0259$ & 0.0248 \\
$\mu_{a}\left(\lambda_{e m}\right)=0.025 \mathrm{~mm}^{-1}$ & $0.0406-0.0412$ & 0.0395 \\
$\mu_{s}\left(\lambda_{e x}\right)=5 \mathrm{~mm}^{-1}$ & $0.0336-0.0349$ & 0.0353 \\
$\mu_{s}\left(\lambda_{e m}\right)=10 \mathrm{~mm}^{-1}$ & $0.0360-0.0370$ & 0.0359 \\
$g\left(\lambda_{e x}\right)=0.70, g\left(\lambda_{e m}\right)=0.76$ & $0.0339-0.0347$ & 0.0336
\end{tabular}

*Базовые значения оптических параметров среды: $\mu_{a}\left(\lambda_{e x}\right)=0.2 \mathrm{~mm}^{-1}, \quad \mu_{a}\left(\lambda_{e m}\right)=0.05 \mathrm{~mm}^{-1}, \quad \mu_{s}\left(\lambda_{e x}\right)=10 \mathrm{~mm}^{-1}, \mu_{s}\left(\lambda_{e m}\right)=5 \mathrm{~mm}^{-1}$, $g\left(\lambda_{e x}\right)=0.80, \quad g\left(\lambda_{e m}\right)=0.84, \quad \eta\left(\lambda_{e x}\right)=\eta\left(\lambda_{e m}\right)=1.45, \quad$ эффективный $\quad$ квантовый $\quad$ выход $\quad$ флуоресценции $Q_{f}^{e f f}=0.25, \quad$ где $Q_{f}^{e f f}=Q_{f}\left(\lambda_{e m}\right)\left[\mu_{a, f}\left(\lambda_{e x}\right) / \mu_{a}\left(\lambda_{e x}\right)\right], \mu_{a, f}\left(\lambda_{e x}\right)$ и $Q_{f}\left(\lambda_{e m}\right)$ - показатель поглощения и квантовый выход флуорофора.

ретикулярной дермы (3) и подкожной жировой клетчатки (4). Толщины слоев: $L_{1}=0.10 \mathrm{~mm}, L_{2}=0.18 \mathrm{~mm}$, $L_{3}=1.82 \mathrm{~mm}, L_{4}=2 \mathrm{~mm}$. Считаем, что эпидермис состоит из поглощающей и рассеивающей свет тканиосновы и поглощающего компонента - меланина. Дерма состоит из той же ткани, в которой равномерно и хаотически распределены кровеносные сосуды. Основные поглотители света в де́рме - гемоглобин (в окисленной $\mathrm{HbO}_{2}$ и в восстановленной $\mathrm{HHb}$ формах), билирубин (Bil) и бета-каротин $(\beta)$. Показатели поглощения эпидермиса и дермы рассчитываются по формулам

$$
\begin{aligned}
\mu_{a}(\lambda)= & f_{m} \mu_{a, m}(\lambda)+\alpha_{V} f_{V} \mu_{a, b l}(\lambda)+C_{\mathrm{tis}, \mathrm{Bil}} \varepsilon_{\mathrm{Bil}}(\lambda) \\
& +C_{\beta} \varepsilon_{\beta}(\lambda)+\left(1-f_{m}-f_{V}\right) \mu_{a, \mathrm{tis}}(\lambda),
\end{aligned}
$$

$\mu_{a, b l}(\lambda)=C_{\mathrm{Hb}}\left(S \varepsilon_{\mathrm{HbO}_{2}}(\lambda)+(1-S) \varepsilon_{\mathrm{HHb}}(\lambda)\right)+C_{\mathrm{bl}, \mathrm{Bil}} \varepsilon_{\mathrm{Bil}}(\lambda)$,

где $f_{m}-$ объемная концентрация меланина (выбранное значение - 2.5\% для эпидермиса, $0 \%$ для дермы), $\mu_{a, m}$ - объемный показатель поглощения меланина [44], $f_{V}$ - объемная концентрация кровеносных сосудов $(0 \%$ для эпидермиса, $3.6 \%$ для папиллярной и $2.7 \%$ для ретикулярной дермы), $\alpha_{V}=\left(1-\exp \left(-\mu_{a, b l} D_{V}\right)\right) /\left(\mu_{a, b l} D_{V}\right)-$ коэффициент коррекции поглощательной способности среды на эффект локализации крови в сосудах (эффект „сита“) [45], $D_{V}$ - диаметр сосудов $(40 \mu \mathrm{m}), \mu_{a, b l}$ - объемный показатель поглощения крови, $C_{\mathrm{Hb}}-$ молярная концентрация гемоглобина в крови $(2.3 \mathrm{mmol} / \mathrm{L}), S$ - степень оксигенации крови $(75 \%), \varepsilon_{\mathrm{HbO}_{2}}$ и $\varepsilon_{\mathrm{HHb}}-$ молярные показатели поглощения $\mathrm{HbO}_{2}$ и $\mathrm{HHb}$ [46], $C_{b l, B i l}$ и $C_{t i s, B i l}$ - молярные концентрации билирубина в крови $(10 \mu \mathrm{mol} / \mathrm{L})$ и в ткани $(2 \mu \mathrm{mol} / \mathrm{L}), \varepsilon_{B i l}-$ молярный показатель поглощения билирубина [47], $C_{\beta}$ - молярная концентрация бета-каротина в ткани $(1 \mu \mathrm{mol} / \mathrm{L}), \varepsilon_{\beta}$ молярный показатель поглощения бета-каротина [48], $\mu_{a, t i s}$ - объемный показатель поглощения соединительной ткани [49]. Транспортные показатели рассеяния $\mu_{s}^{\prime}$ эпидермиса и дермы полагаются одинаковыми
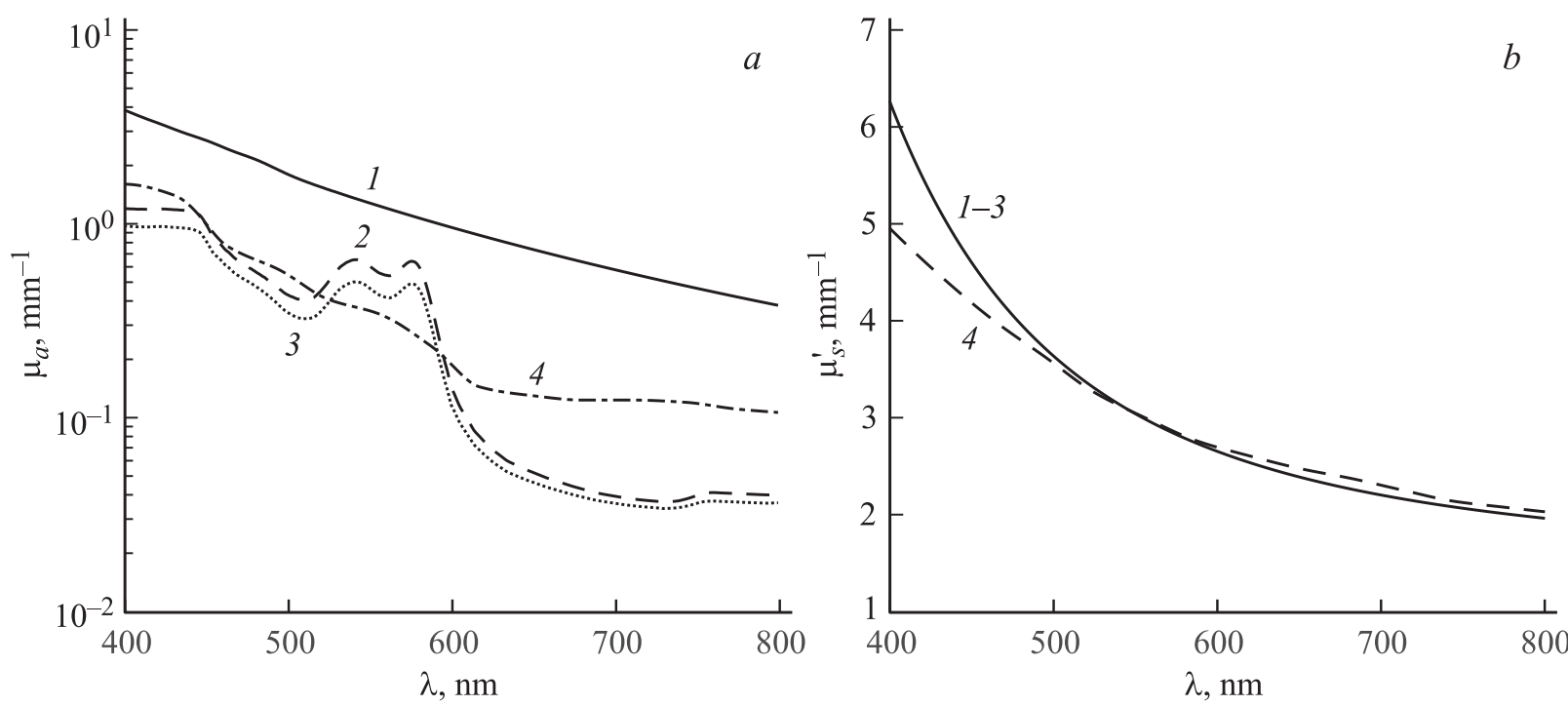

Рис. 2. Спектральные зависимости показателей поглощения $(a)$ и транспортных показателей рассеяния $(b)$ четырех слоев кожи: эпидермиса (1), папиллярной дермы (2), ретикулярной дермы (3) и жировой клетчатки (4). 

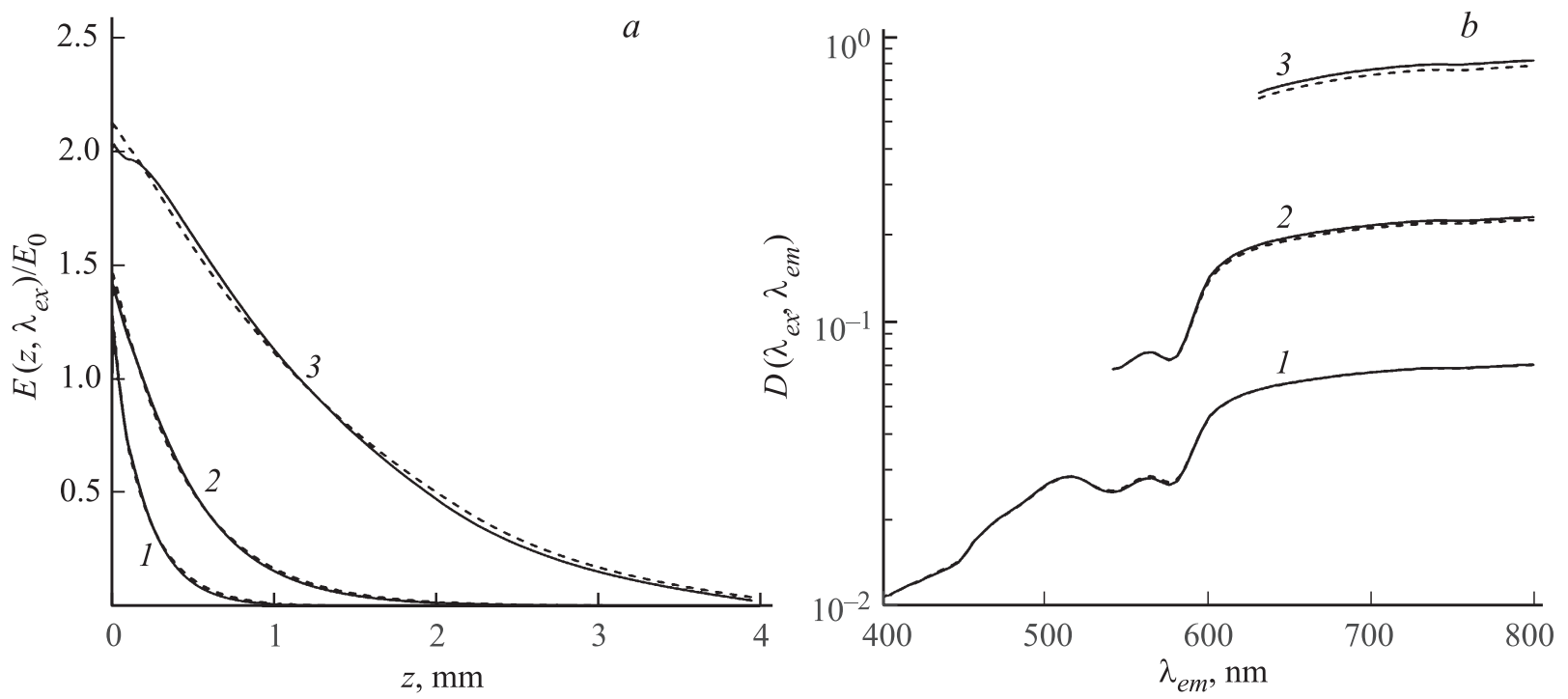

Рис. 3. Сопоставление характеристик переноса излучения в коже, смоделированных методом Монте-Карло (сплошные линии) и рассчитанных полуаналитическим методом (штриховые линии): распределения потока возбуждающего излучения по глубине кожи $(a)$ и передаточные функции кожи $(b)$ для спектров флуоресценции, регистрируемых с ее поверхности, при $\lambda_{e x}=400(1)$, 540 (2) и $630(3) \mathrm{nm}$.

и соответствующими экспериментальным данным [50]. Для моделирования спектральных зависимостей показателей $\mu_{a}$ и $\mu_{s}^{\prime}$ жировой клетчатки используются данные работы [51]. Средний косинус индикатрисы рассеяния $g$ всех слоев кожи рассчитывается по эмпирической формуле: $g=0.62+0.29 \lambda \cdot 10^{-3}[52]$, где $\lambda$ выражена в $\mathrm{nm}$. Показатель преломления $\eta$ всех слоев кожи полагается равным 1.45. Результирующие оптические параметры среды, моделирующей кожу человека, представлены в зависимости от $\lambda$ на рис. 2 .

Полагаем, что флуоресценция возникает только в тканях дермы (на глубине $0.1-2.0 \mathrm{~mm}$ ) в результате возбуждения одного флуорофора, равномерно распределенного по ее толщине. Передаточная функция среды $D\left(\lambda_{e x}, \lambda_{e m}\right)$, связывающая регистрируемый спектр флуоресценции $F\left(\lambda_{e x}, \lambda_{e m}\right)$ с истинным спектром свечения флуорофора $X_{f}\left(\lambda_{e x}, \lambda_{e m}\right)$, определяется следующим выражением:

$$
D\left(\lambda_{e x}, \lambda_{e m}\right)=\frac{F\left(\lambda_{e x}, \lambda_{e m}\right)}{E_{0}\left(\lambda_{e x}\right) X_{f}\left(\lambda_{e x}, \lambda_{e m}\right)},
$$

где $E_{0}-$ освещенность среды, $X_{f}\left(\lambda_{e x}, \lambda_{e m}\right)=$ $=C_{f} \varepsilon_{f}\left(\lambda_{e x}\right) Q_{f}\left(\lambda_{e m}\right)$.

Моделирование переноса излучения в исследуемой среде выполняется методом МК в следующей последовательности. На первом этапе моделируется световое поле в среде на длине волны возбуждения флуоресценции $\lambda_{e x}$. Эта задача решалась на основе рассмотрения $10^{6}$ случайных траекторий фотонов. Алгоритм моделирования траектории каждого фотона подробно описан во многих работах, например в $[9,10]$. Световой поток на глубине $z$ рассчитывается путем суммирования „весов“ всех фотонов, пролетающих через заданную глубину во всех направлениях. На втором этапе моделирования рассматриваются траектории фотонов, испущенных в результате флуоресценции внутри среды. Глубина испускания фотона разыгрывается в соответствии с распределением концентрации флуорофора $C_{f}(z)$ и потока возбуждающего излучения $E\left(z, \lambda_{e x}\right)$ в среде. Для этого генерируется случайное число $\xi$ из диапазона $[0,1]$ и решается следующее уравнение:

$$
P(z)=\frac{\int_{z_{1}}^{z} C_{f}(z) E\left(z, \lambda_{e x}\right) d z}{\int_{z_{1}}^{z_{2}} C_{f}(z) E\left(z, \lambda_{e x}\right) d z}=\xi,
$$

где $P(z)$ - вероятность возникновения фотона на глубине $z, z_{1}$ и $z_{2}$ - границы слоя среды с флуорофором. При дискретном задании функции $P(z)$ и ее линейной интерполяции в интервалах между узлами дискретизации $\left(z_{k}, z_{k+1}\right)$ уравнение (18) сводится к квадратному уравнению, решение которого имеет следующий вид:

$$
z=z_{k}+\frac{\sqrt{P^{2}\left(z_{k}\right)+2 \Delta_{k}\left(\xi-X_{k}\right)}-P\left(z_{k}\right)}{\Delta_{k}},
$$

где

$$
\begin{gathered}
X_{k}=\frac{1}{2} \sum_{i=1}^{k-1}\left(P\left(z_{i+1}\right)+P\left(z_{i}\right)\right)\left(z_{i+1}-z_{i}\right), \\
\Delta_{k}=\left(P\left(z_{k+1}\right)-P\left(z_{k}\right)\right) /\left(z_{k+1}-z_{k}\right) .
\end{gathered}
$$

Процессы поглощения, рассеяния и свободного пробега фотона в среде моделируются до тех пор, пока „вес“ фотона не уменьшится в $10^{4}$ раз по сравнению с его первоначальным значением, либо пока фотон не вылетит из среды. В качестве индикатрисы рассеяния 
среды используется функция Хеньи-Гринштейна с единственным параметром - средним косинусом $g$. Отражение и преломление фотонов на границе раздела сред описывается законами геометрической оптики и формулами Френеля. Поток флуоресценции с поверхности среды рассчитывается как отношение суммарного „веса“ фотонов, сгенерированных внутри среды, долетевших до ее поверхности и вылетевших наружу, к суммарному „весу“ фотонов, введенных в среду.

Результаты расчетов характеристик переноса излучения в четырехслойной среде, моделирующей кожу человека, приведены на рис. 3. Расчеты выполнены для трех случаев, соответствующих облучению среды на $\lambda_{e x}=400,540,630 \mathrm{~nm}$ и ее флуоресценции в области $\lambda_{e x} \leq \lambda_{e m} \leq 800 \mathrm{~nm}$. Видно, что распределения светового потока по глубине среды, полученные разработанным методом, хорошо согласуются с результатами их моделирования методом МК как при сильном (на $\lambda_{e x}=400 \mathrm{~nm}$ ), так и при слабом (на $\lambda_{e x}=630 \mathrm{~nm}$ ) поглощении света в среде. Различие передаточных функций среды (17), рассчитанных двумя методами, не превышает $2.4 \%$ при $\lambda_{e x}=400 \mathrm{~nm}, 2.6 \%$ при $\lambda_{e x}=540 \mathrm{~nm}$ и $4.5 \%$ при $\lambda_{e x}=630 \mathrm{~nm}$. Таким образом, разработанный метод представляет хорошую альтернативу времязатратному методу МК в задачах расчета спектров флуоресценции многослойных оптически плотных сред.

\section{Коррекция спектров флуоресценции}

Результаты моделирования передаточных функций кожи, представленные на рис. $3, b$, демонстрируют сходство функции $D\left(\lambda_{e x}, \lambda_{e m}\right)$ со спектром КДО кожи, что обусловливает перспективность использования спектроскопии диффузного отражения для коррекции сигналов флуоресценции биотканей на эффекты поглощения и рассеяния в них света. Данные об оптических параметpax среды, необходимые для расчета корректирующей функции (17), можно извлекать из спектра КДО среды, измеряемого тем же прибором, что и спектр флуоресценции. Для решения соответствующей обратной задачи необходима модель КДО исследуемой среды, позволяющая при сравнительно небольшом количестве свободных параметров адекватно воспроизводить результаты спектральных измерений. Одна из таких моделей была предложена для кожи человека в работе [36]. Модель основана на высокоточном аппроксимационном аналоге метода МК для двухслойной среды и позволяет в явном виде рассчитывать КДО кожи в зависимости от ее показателя преломления, толщины эпидермиса, показателей поглощения и рассеяния эпидермиса и дермы (последняя представлена однородным полубесконечным слоем). Эта модель в сочетании с описанным выше методом решения одномерной задачи флуоресценции может достаточно эффективно использоваться для определения концентраций флуорофоров в коже на основе совместных измерений спектров ее отражения и флуо- ресценции. Продемонстрируем это на конкретном примере - коррекции спектра флуоресценции 5-АЛК-индуцированного ПП IX, регистрируемого с поверхности кожи человека.

5-аминолевулиновая кислота (5-АЛК) - это биогенная кислота, являющаяся одним из промежуточных продуктов синтеза гема. При ее введении в организм (системно или местно) она приводит к остановке синтеза гема в опухолевых клетках на этапе образования протопорфирина IX и его интенсивному накоплению в опухоли. ПП IX является эффективным эндогенным флуорофором для визуализации опухолей и фотосенсибилизатором для фотодинамической терапии. Он имеет максимум поглощения на $\lambda_{e x}=405 \mathrm{~nm}$ и флуоресцирует в красной области спектра с максимумами на $\lambda_{e m}=635$ и $705 \mathrm{~nm}$ [35]. Для стандартизации методов флуоресцентной диагностики и фотодинамической терапии с использованием 5-АЛК-индуцированного ПП IX, очевидно, необходимо иметь возможность количественного определения его концентрации в ткани пациента. В связи с этим оценим влияние среды распространения излучения на спектр флуоресценции ПП IX, регистрируемый с поверхности кожи человека, и эффективность коррекции искажений спектра флуоресценции ПП IX с использованием измерений КДО кожи и разработанных методов теории переноса излучения.

В качестве тестовых спектров отражения и флуоресценции кожи будем использовать результаты их моделирования методом МК в рамках описанной выше модели четырехслойной среды, достаточно детально учитывающей анатомическое строение кожи. Моделирование проведено при трех значениях параметров среды, соответствующих различной пигментации кожи. В первом случае используются указанные выше значения параметров среды, типичные для нормальной светлой кожи человека. Два других случая отличаются от первого повышенными содержаниями меланина в эпидермисе и кровеносных сосудов в де́рме. Тестовые спектры диффузного отражения и флуоресценции фантома кожи приведены на рис. $4, a, b$. Видно, что спектры флуоресценции ПП IX, смоделированные при различной пигментации кожи, схожи по форме, но существенно различаются по величине сигнала, что при отсутствии их должной коррекции может приводить к ошибочной интерпретации результатов измерений. В частности, увеличение интенсивности флуоресценции за счет накопления ПП IX в опухолевой ткани может не проявляться визуально при аномально высокой репродукции меланоцитов (клеток-производителей меланина), характерной для меланомы, а также и при разрастании кровеносной сети опухоли (при ангиогенезе).

Коррекция спектров флуоресценции кожи выполняется в следующей последовательности. Вначале решается обратная задача для спектра диффузного отражения кожи с использованием его аналитической модели $[36,53]$. Искомыми параметрами модели являются: толщина эпидермиса $L_{e p}$, объемная концентрация меланина в эпидер- 

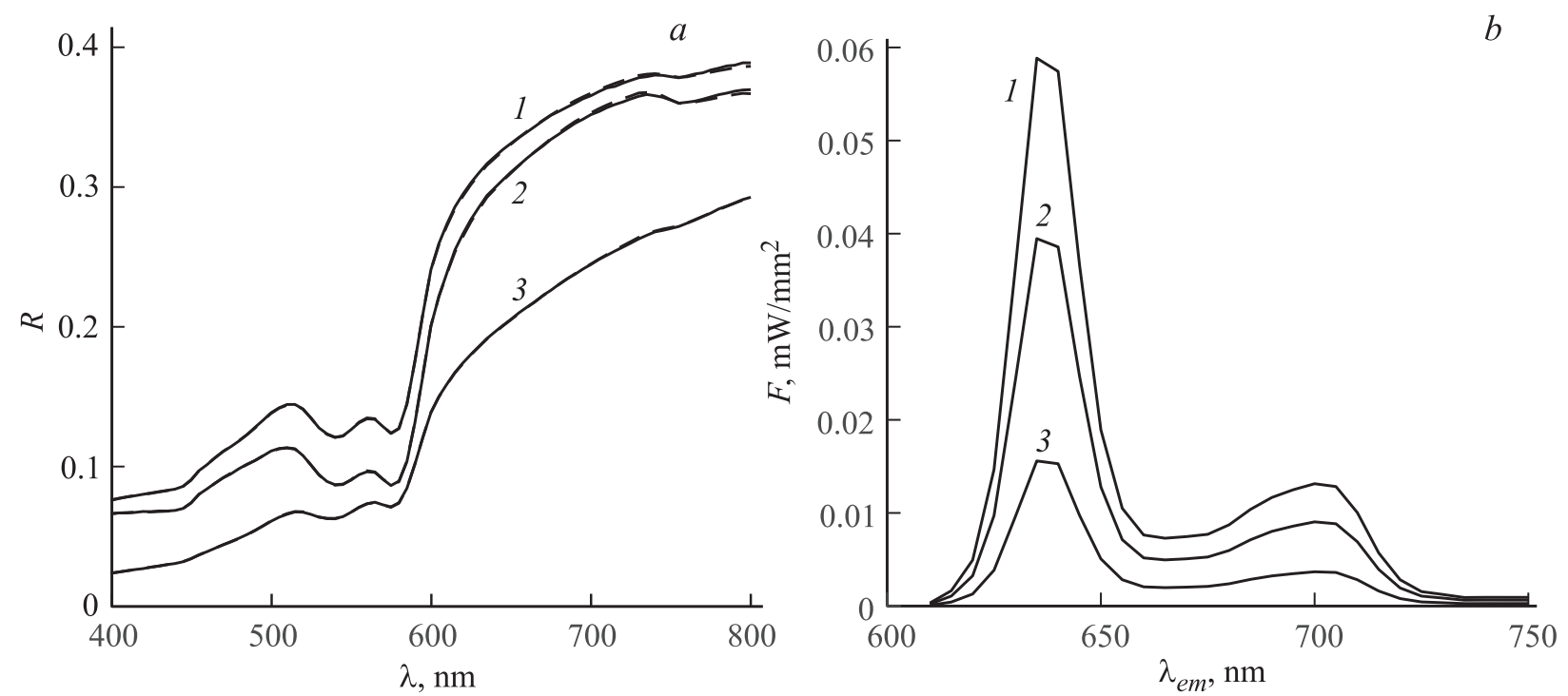

Рис. 4. Спектры диффузного отражения $(a)$ и флуоресценции $(b)$ четырехслойного фантома кожи с содержаниями меланина в эпидермисе $2.5 \%(1,2)$ и $7 \%(3)$, кровеносных сосудов в папиллярной дерме $3.6 \%(1,3)$ и $7 \%(2)$, кровеносных сосудов в ретикулярной дерме $2.7 \%(1,3)$ и $5 \%(2)$; штриховые линии - результаты подбора спектров диффузного отражения среды в рамках аналитической модели кожи.
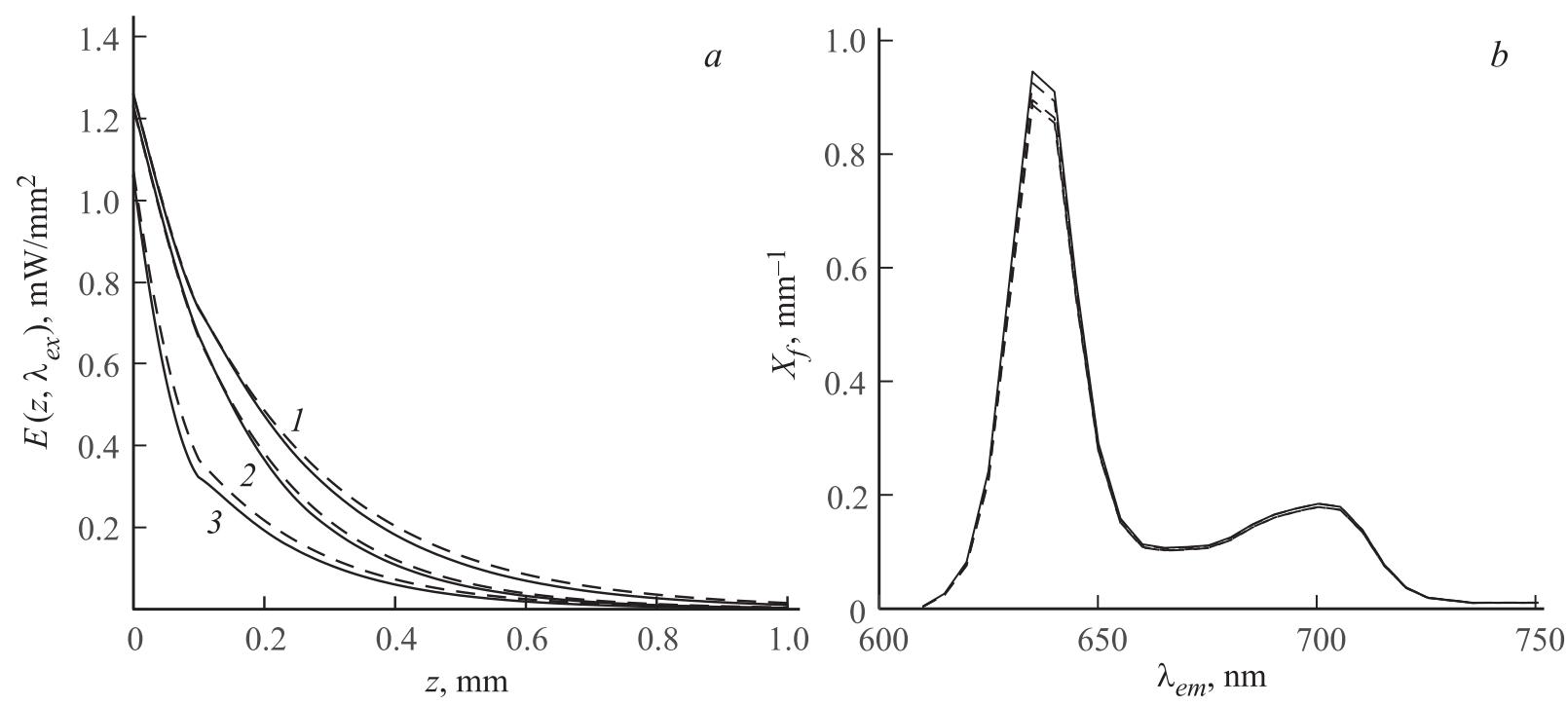

Рис. 5. Распределения светового потока на $\lambda_{e x}=405 \mathrm{~nm}(a)$ и спектры флуоресценции протопорфирина IX $(b)$ внутри четырехслойного фантома кожи: линии - фактические зависимости, штрихи - результаты решения обратной задачи в рамках оптической модели двухслойной среды „эпидермис-дерма“; цифры соответствуют различным содержаниями меланина и кровеносных сосудов в коже (аналогично рис. 4).

мисе $f_{m}$, объемная концентрация кровеносных сосудов в однородной и полубесконечной дерме $f_{V}$, диаметр сосудов $D_{V}$, степень оксигенации крови $S$, молярная концентрация билирубина в крови $C_{b l, B i l}$ (концентрация билирубина в окружающей ткани полагается равной $\left.0.2 C_{b l, B i l}\right)$, молярная концентрация бета-каротина в дерме $C_{\beta}$, параметры $\left(C_{s}, \nu, \gamma\right)$ спектральной зависимости транспортного показателя рассеяния ткани эпидермиса и дермы вида $\mu_{s}^{\prime}(\lambda)=C_{s}\left[\gamma\left(\lambda_{0} / \lambda\right)^{v}+(1-\gamma)\left(\lambda_{0} / \lambda\right)^{4}\right]$ (суперпозиция спектров рассеяния Ми и Релея), пока- затель преломления кожи $\eta$. Спектральные зависимости показателей поглощения эпидермиса $\mu_{a, e p}$ и дер-

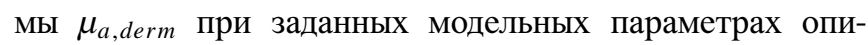
сываются формулами (15), (16). КДО кожи рассчитывается аналитически в зависимости от ее оптических параметров $\left(\mu_{s}^{\prime}, \mu_{a, e p}, \mu_{a, d e r m}, \eta\right)$ и толщины верхнего слоя $L_{e p}$ (эпидермиса). Учет при решении обратной задачи только двух слоев кожи связан с малой глубиной проникновения в нее света, возбуждающего флуоресценцию ПП IX $(405 \mathrm{~nm})$. Кроме того, объединение 
анатомических областей дермы в один однородный слой улучшает постановку обратной задачи за счет уменьшения количества неизвестных параметров кожи, одинаково влияющих на ее спектр диффузного отражения. Параметры модели, обеспечивающие минимальную невязку между измеряемыми и аналитическими спектрами КДО кожи, подбираются по итерационной формуле Левенберга-Марквардта [54]. Исходные и подобранные спектры четырехслойного фантома кожи сопоставлены на рис. 4, a. Максимальное различие между ними не превышает $1 \%$.

На втором этапе коррекции спектра флуоресценции кожи рассчитывается распределение светового потока $E\left(z, \lambda_{e x}\right)$ по формулам (2)-(9) при значениях оптических параметров кожи, восстановленных из ее спектра КДО. Фактические распределения $E\left(z, \lambda_{e x}\right)$ в четырехслойных фантомах кожи с различной пигментацией и соответствующие распределения, рассчитанные в приближении кожи двухслойной средой, сопоставлены на рис. 5, $a$. Хорошее согласие между представленными распределениями свидетельствует в пользу использования упрощенной (двухслойной) модели для решения прямой и обратной задач переноса излучения в коже.

Данные об оптических параметрах кожи в спектральной области ее флуоресценции и распределении в ней потока возбуждающего излучения позволяют рассчитать передаточную функцию (17) и скорректировать искажения измеряемого спектра флуоресценции, вызванные средой распространения излучения. Примеры такой коррекции приведены на рис. $5, b$. Видно, что скорректированные спектры практически полностью воспроизводят истинный спектр флуоресценции ПП IX в четырехслойном фантоме кожи. Наибольшая погрешность коррекции тестовых спектров получена при высоком содержании кровеносных сосудов в среде, моделирующей кожу, и составляет $\sim 8 \%$.

\section{Заключение}

Разработанный метод расчета потока вынужденной флуоресценции многослойной оптически плотной среды с произвольным распределением в ней флуорофора позволяет без существенных для практики вычислительных затрат решать прямые и обратные задачи флуоресцентной спектроскопии биотканей как при сильном, так и при слабом поглощении в них света. Различия между результатами решения прямой задачи флуоресценции для кожи человека (задачи расчета потока флуоресценции при известных значениях оптических параметров среды и концентрации в ней флуорофора) разработанным методом и методом МК находятся на уровне $1 \%$. Предложенный метод в сочетании с аппроксимационноаналитическими моделями диффузного отражения биотканей $[36,43,53]$ формируют надежную методическую основу для количественного анализа спектров отражения и флуоресценции биотканей, а также для кор- рекции измеряемых сигналов флуоресценции на влияние среды распространения излучения. Возможность такой коррекции важна для определения концентраций флуоресцентных онкомаркеров в новообразованиях и поиска объективных диагностических признаков злокачественных опухолей. Кроме того, большинство фотосенсибилизаторов для фотодинамической терапии рака флуоресцируют в красной области спектра, что делает перспективным использование предложенных методик для решения задач дозиметрии лазерного излучения.

\section{Список литературы}

[1] Tuchin V.V. Tissue Optics: Light Scattering Methods and Instruments for Medical Diagnosis. Bellingham: SPIE Press, USA, 2007. $882 \mathrm{p}$.

[2] Тучин В.В. Лазеры и волоконная оптика в биомедицинских исследованиях. М.: Физматлит, 2010. 478 с.

[3] Ramanujam N. // Encyclopedia of Analytical Chemistry / Ed. by Meyers R. Chichester: John Wiley and Sons, 2000. P. 20 56. doi 10.1002/9780470027318.a0102.

[4] Maynard J.D., Rohrscheib M., Way J.F., Nguyen C.M., Ediger M.N. // Diabetes Care. 2007. V. 30. P. 1120.

[5] Kang Uk., Папаян Г.В., Березин В.Б., Петрищев Н.Н., Галагудза М.М. // Оптический журнал. 2013. Т. 80. № 1. C. 56.

[6] Bradley R.S., Thorniley M.S. // J. R. Soc. Interface. 2006. V. 3. № 6. P. 1. doi 10.1098/rsif.2005.0066.

[7] Hull E., Ediger M., Unione A., Deemer E., Stroman M., Baynes J. // Opt. Express. 2004. V. 12. N 19. P. 4496. doi 10.1364/OPEX.12.004496.

[8] Weersink R., Patterson M.S., Diamond K., Silver S., Padgett N. // Appl. Opt. 2001. V. 40. N 34. P. 6389. doi 10.1364/AO.40.006389.

[9] Wang L.H., Jacques S.L., Zheng L.Q. // Comput. Methods Programs Biomed. 1995. V. 47. N 2. P. 131. doi 10.1016/0169-2607(95)01640-F.

[10] Welch A.J., Gardner C., Richards-Kortum R., Chan E., Criswell G., Pfefer J., Warren S. // Lasers. Surg. Med. 1997. V. 21. N 2. P. 166. doi 10.1002/(SICI)1096-9101(1997)21:23.3.CO;2-Q.

[11] Qu J.Y., MacAulay C.E., Lam S., Palcic B. // Opt. Eng. 1995. V. 34. N 11. P. 3334. doi 10.1117/12.212917.

[12] Zonios G.I., Cothren R.M., Arendt J.T., Wu J., Van Dam J., Crawford J.M., Manoharan R., Feld M.S. // IEEE Trans. Biomed. Eng. 1996. V. 43. N 2. P. 113. doi 10.1109/10.481980.

[13] Drezek R., Sokolov K., Utzinger U., Boiko I., Malpica A., Follen M., Richards-Kortum R. // J. Biomed. Opt. 2001. V. 6. N 4. P. 385. doi 10.1117/1.1413209.

[14] Liu C., Rajaram N., Vishwanath K., Jiang T., Palmer G.M., Ramanujam N. // J. Biomed. Opt. 2012. V. 17. N 7. P. 077012. doi 10.1117/1.JBO.17.7.077012.

[15] Кузнецова А.А., Пушкарева А.Е. // Оптика и спектр. 2016. T. 121. № 2. C. 328; Kuznetsova A.A., Pushkareva A.E. // Opt. Spectrosc. 2016. V. 121. N 2. P. 304.

[16] Дрёмин В.В., Дунаев А.В. // Оптический журнал. 2016. T. 83. № 1. C. 57.

[17] Müller M.G., Georgakoudi I., Zhang Q., Wu J., Feld M.S. // Appl. Opt. 2001. V. 40. N 25. P. 4633. doi 10.1364/AO.40.004633. 
[18] Du Le V.N., Patterson M.S., Farrell T.J., Hayward J.E., Fang Q. // J. Biomed. Opt. 2015. V. 20. N 12. P. 127003. doi 10.1117/1.JBO.20.12.127003.

[19] Sinichkin Yu.P., Utz S.R., Mavliutov A.H., Pilipenko H.A. // J. Biomed. Opt. 1998. V. 3. N 2. P. 201.

[20] Lin W.C., Toms S.A., Jansen E.D., Mahadevan-Jansen A. // IEEE J. Sel. Top. Quant. 2001. V. 7. N 6. P. 996. doi 10.1109/2944.983305.

[21] Kokhanovsky A.A. // J. Opt. Soc. Am. A. 2009. V. 26. N 8. P. 1896. doi 10.1364/JOSAA.26.001896.

[22] Рогаткин Д.А., Смирнова О.Д. // Оптический журнал. 2013. T. 80. № 9. C. 54.

[23] Zhadin N.N., Alfano R.R. // J. Biomed. Opt. 1998. V. 3. N 2. P. 171. doi 10.1117/1.429874.

[24] Hyde D.E., Farrell T.J., Patterson M.S., Wilson B.C. // Phys. Med. Biol. 2001. V. 46. N 2. P. 369.

[25] Diamond K.R., Farrell T.J., Patterson M.S. // Phys. Med. Biol. 2003. V. 48. N 24. P. 4135.

[26] Kim A., Khurana M., Moriyama Y., Wilson B.C. // J. Biomed. Opt. 2010. V. 15. N 6. P. 067006. doi 10.1117/1.3523616.

[27] Chang S.K., Arifler D., Drezek R., Follen M., RichardsKortum R. // J. Biomed. Opt. 2004. V. 9. N 3. P. 511. doi 10.1117/1.1695559.

[28] Gardner C.M., Jacques S.L., Welch A.J. // Lasers Surg. Med. 1996. V. 18. N 2. P. 129. doi 10.1002/(SICI)1096-9101(1996)18:2<129::AID-LSM2>3.0.CO;2-U.

[29] Kanick S.C., Robinson D.J., Sterenborg H.J., Amelink A. // Opt. Lett. 2012. V. 37. N 5. P. 948. doi 10.1364/OL.37.000948.

[30] Yudovsky D., Pilon L. // Appl. Opt. V. 49. N 31. P. 6072. doi 10.1364/AO.49.006072.

[31] Ishimaru A. Wave Propagation and Scattering in Random Media. V. 1: Single Scattering and Transport Theory. San Diego, California: Academic Press, 1978. 272 р.; Исимаpy $A$. Распространение и рассеяние волн в случайнонеоднородных средах. Т. 1. М.: Мир, 1981. 285 с.

[32] Лысенко С.А., Кугейко М.М. // Журн. прикл. спектр. 2013. T. 80. № 2. C. 273; Lisenko S.A., Kugeiko M.M. // J. Appl. Spectr. 2013. V. 80. N 2. P. 271. doi 10.1007/s10812-013-9757-9.

[33] Лысенко С.А., Кугейко М.М. // Журн. прикл. спектр. 2014. T. 81. № 5. C. 761; Lisenko S.A., Kugeiko M.M. // J. Appl. Spectr. 2014. V. 81. N 5. P. 834. doi 10.1007/s10812-014-0013-8.

[34] Лысенко С.А., Кугейко М.М. // Квант. электрон. 2015. T. 45. № 4. C. 358; Lisenko S.A., Kugeiko M.M. // Quantum. Electron. 2015. V. 45. N 4. P. 358. doi 10.1070/QE2015v045n04ABEH015381.

[35] Rollakanti K.R., Kanick S.C., Davis S.C., Pogue B.W., Maytin E.V. // Photon. Lasers Med. 2013. V. 2. N 4. P. 287. doi 10.1515/plm-2013-0030.

[36] Лысенко С.А., Кугейко М.М. // Квант. электрон. 2014. T. 44. № 3. C. 252; Lisenko S.A., Kugeiko M.M. // Quantum. Electron. 2014. V. 44. N 3. P. 252. doi 10.1070/QE2014v044n03ABEH015339.

[37] Balas C. // IEEE Trans. Biomed. Eng. 2001. V. 48. N 1. P. 96. doi 10.1109/10.900259.

[38] Tran C.D. // Anal. Lett. 2005. V. 38. N 5. P. 735. doi 10.1081/AL-200047754.

[39] Graaff R., Meerwaldt R., Lutgers H.L., Baptist R., de Jong E.D., Zijp J.R., Links T.P., Smit A.J., Rakhorst G. // Proc. SPIE. 2005. V. 5692. P. 111. doi 10.1117/12.588984.
[40] Зеге Э.П., Бушимакова О.Е., Кацев И.Л., Коновалов Н.В. // Журн. прикл. спектр. 1979. Т. 30. № 5. С. 900.

[41] Liou K.N. An introduction to atmospheric radiation. Second edition. N. Y., London: Academic Press, 2002. 583 p.

[42] Swartling J., Pifferi A., Enejder A.M., Andersson-Engels S. // J. Opt. Soc. Am. A. 2003. V. 20. N 4. P. 714. doi 10.1364/JOSAA.20.000714.

[43] Лысенко С.А., Кугейко М.М. // Измерит. техн. 2013. № 11. C. 68; Lysenko S.A., Kugeiko M.M. // Meas. Tech. 2014. V. 56. N 11. P. 1302. doi 10.1007/s11018-014-0372-9.

[44] Jacques S.L. Melanosome absorption coefficient. [Электронный ресурс] Режим доступа: http://omlc.org/spectra/melanin/mua.html

[45] Verkruysse W., Lucassen G.W., de Boer J.F., Smithies D.J., Nelson J.S., Van Gemert M.J.C. // Phys. Med. Biol. 1997. V. 42. N 1. P. 51.

[46] Prahl S.A. Optical Absorption of Hemoglobin. [Электронный pecypc] Режим доступа: http://omlc.org/spectra/hemoglobin/

[47] Prahl S.A. Bilirubin. [Электронный ресурс] Режим доступа: http://omlc.org/spectra/PhotochemCAD/html/119.html

[48] Prahl S.A. Beta-carotene. [Электронный pecypc] Режим доступа: http://omlc.org/spectra/PhotochemCAD/html/041.html

[49] Jacques S.L. Skin Optics. [Электронный pecypc] Режим доступа: http://omlc.org/news/jan98/skinoptics.html

[50] Bashkatov A.N., Genina E.A., Kochubey V.I., Tuchin V.V. // J. Phys. D: Appl. Phys. 2005. V. 38. N 15. P. 2543. doi 10.1088/0022-3727/38/15/004.

[51] Salomatina E., Jiang B., Novak J., Yaroslavsky A.N. // J. Biomed. Opt. 2006. V. 11. N 6. P. 064026. doi 10.1117/1.2398928.

[52] Van Gemert M.J.C., Jacques S.L., Sterenborg H.J.C.M., Star W.M. // IEEE Trans. Biomed. Eng. 1989. V. 36. N 12. P. 1146. doi 10.1109/10.42108.

[53] Лысенко С.А., Кугейко М.М., Фираго В.А., Собчук А.Н. // Квант. электрон. 2014. Т. 44. № 1. С. 69; Lisenko S.A., Kugeiko M.M., Firago V.A., Sobchuk A.N. // Quantum Electron. 2014. V. 44. N 1. P. 69. doi 10.1070/QE2014v044n01ABEH015233.

[54] Press W.H., Teukolsky S.A., Vetterling W.T., Flannery B.P. Numeric recipes. The art of scientific computing. Third edition. N. Y.: Cambridge University Press, 2007. P. 801-806. 